\title{
Exact real-time dynamics of the quantum Rabi model
}

\author{
F. Alexander Wolf, ${ }^{1}$ Marcus Kollar, ${ }^{2}$ and Daniel Braak ${ }^{1}$ \\ ${ }^{1}$ Experimental Physics VI, ${ }^{2}$ Theoretical Physics III, \\ Center for Electronic Correlations and Magnetism, \\ University of Augsburg, 86135 Augsburg, Germany
}

(Dated: April 1, 2012)

\begin{abstract}
We use the analytical solution of the quantum Rabi model to obtain absolutely convergent series expressions of the exact eigenstates and their scalar products with Fock states. This enables us to calculate the numerically exact time evolution of $\left\langle\sigma_{x}(t)\right\rangle$ and $\left\langle\sigma_{z}(t)\right\rangle$ for all regimes of the coupling strength, without truncation of the Hilbert space. We find a qualitatively different behavior of both observables which can be related to their representations in the invariant parity subspaces.

PACS numbers: 03.65.Ge,02.30.Ik,42.50.Pq
\end{abstract}

\section{INTRODUCTION}

The quantum Rabi model (QRM) describes one of the simplest strongly coupled quantum systems: a single bosonic mode interacts with a two-level system. It forms the basic building block of theoretical approaches to the interaction of light with matter [1] or to electron-phonon interaction [2]. Due to recent progress in nanofabrication, the strong-coupling regime of the QRM could be accessed experimentally within circuit QED [3, 4], where the twolevel system corresponds to a single qubit. To construct controllable solid-state quantum gates, it is necessary to comprehend the behavior of the QRM for all regimes of the coupling strength [5].

Although the lower part of the spectrum of the QRM can be computed numerically to arbitrary precision using exact diagonalization on a truncated state space due to the convergence properties of the associated continued fraction [6-9], it is by no means evident whether the properties of the numerically obtained eigenstates correspond to those of the true eigenstates of the untruncated model. Even if the spectra of the truncated and the full model are close for a subset of the eigenvalues, the corresponding eigenvectors could still be related by a unitary transformation, which does not necessarily approach the identity. Consequently, a variety of systematic approximations making use of different basis sets in the infinitedimensional Hilbert space have been developed $[2,10-15]$. However, all of them implement a truncation procedure at some stage of the calculation and thus cannot answer the basic question on the relation of the truncated to the full model.

Beside this theoretical goal, there is a quite practical reason to investigate the true eigenbasis of the QRM: To compute the dynamics of the system on all time scales, not only the exact eigenvalues, but also the exact eigenstates are required. Only then one may hope to predict reliably the behavior of qubits within a circuit QED setup [5].

In this paper we solve the problem by means of the recently obtained exact solution of the QRM [16]. We construct absolutely convergent series expansions of the eigenstates in terms of known basis sets, which allows to estimate the true error in any numerical evaluation. It turns out that a standard calculation with double precision, sufficient to compute the spectrum, fails for the eigenstates.

The QRM Hamiltonian reads

$$
H_{\mathrm{R}}=\omega a^{\dagger} a+g \sigma_{x}\left(a+a^{\dagger}\right)+\frac{\omega_{0}}{2} \sigma_{z} .
$$

The Hilbert space is given by $L^{2}(\mathbb{R}) \otimes \mathbb{C}^{2}$, where the basis of the spin part of the wavefunction $|\sigma\rangle$ is $\{|+1\rangle,|-1\rangle\}$. We employ the Bargmann space $\mathcal{B}$ of analytical functions to represent elements of $L^{2}(\mathbb{R})$ [17]. In $\mathcal{B}$ the creation (annihilation) operators $a^{\dagger}(a)$ are represented as $z\left(\partial_{z}\right)$ and normalization is defined with respect to the scalar product

$$
\langle\psi \mid \phi\rangle=\frac{1}{\pi} \int \mathrm{d} z \mathrm{~d} \bar{z} e^{-z \bar{z}} \overline{\psi(z)} \phi(z) .
$$

An arbitrary analytical function $\phi(z)$ is an element of the Bargmann space $\mathcal{B}$ if $\langle\phi \mid \phi\rangle<\infty$.

Each eigenstate of $H_{\mathrm{R}}$ belongs to one invariant subspace (parity chain) [18] with a fixed $\mathbb{Z}_{2}$ quantum number, the parity, taking values \pm 1 . The parity operator for $H_{\mathrm{R}}$ is defined as $\hat{P}=(-1)^{z \partial_{z}} \sigma_{z}$. Due to $\left[H_{\mathrm{R}}, \hat{P}\right]=0$, the Hilbert space is a direct sum of invariant subspaces $\mathcal{H}_{+} \oplus \mathcal{H}_{-}$and $H_{\mathrm{R}}$ takes the form $H_{ \pm}$on $\mathcal{H}_{ \pm}$.

The invariant parity chains $\mathcal{H}_{ \pm}$are spanned by $\left\{\phi_{s}(z) \otimes| \pm 1\rangle, \phi_{a}(z) \otimes|\mp 1\rangle\right\}$, where $\phi_{a, s}=(1 / 2)[\phi(z) \mp$ $\phi(-z)]$ is the (anti-)symmetric part of $\phi(z)$. Each parity chain is therefore isomorphic to $\mathcal{B}$ via the mapping

$$
\phi(z) \leftrightarrow \phi_{s}(z) \otimes| \pm 1\rangle+\phi_{a}(z) \otimes|\mp 1\rangle,
$$

where $\phi_{s}(z) \otimes| \pm 1\rangle+\phi_{a}(z) \otimes|\mp 1\rangle=|\phi, \pm\rangle$ is an element of $\mathcal{H}_{ \pm}$. Using this isomorphism, the Hamiltonian $H_{ \pm}$ reads

$$
H_{ \pm}=\omega z \partial_{z}+g\left(z+\partial_{z}\right) \pm \Delta \hat{T}
$$

with $\Delta=\omega_{0} / 2$, and the reflection operator $\hat{T}$ acts on elements of $\mathcal{B}$ as $\hat{T}(\phi)(z)=\phi(-z)$. For notational simplicity, we restrict ourselves to $\mathcal{H}_{+}$and measure all energies in units of $\omega$. 
The eigenfunctions $\psi_{m}(z)$ and eigenvalues $E_{m}$ of $H_{+}$ have been obtained in Ref. 16 as two equivalent representations,

$$
\begin{aligned}
& \psi_{m}(z)=\phi_{2}\left(x_{m},-z\right)=e^{g z} \sum_{n=0}^{\infty} K_{n}\left(x_{m}\right)(-z+g)^{n} \\
& \psi_{m}(z)=\phi_{1}\left(x_{m}, z\right)=e^{-g z} \sum_{n=0}^{\infty} K_{n}\left(x_{m}\right) \Delta \frac{(z+g)^{n}}{x_{m}-n}
\end{aligned}
$$

where $x_{m}=E_{m}+g^{2}$ and

$$
\begin{aligned}
n K_{n}(x) & =f_{n-1}(x) K_{n-1}(x)-K_{n-2}(x), \\
K_{1}(x) & =f_{0}(x), \quad K_{0}(x)=1, \\
f_{n}(x) & =\frac{2 g}{\omega}+\frac{1}{2 g}\left(n \omega-x+\frac{\Delta^{2}}{x-n \omega}\right) .
\end{aligned}
$$

Both (5a) and (5b) converge for all $z$ in the complex plane if and only if $x_{m}$ satisfies the spectral condition [16]

$$
\begin{aligned}
& G_{+}\left(x_{m}, 0\right)=0 \quad \text { where } \\
& G_{+}(x, z)=\phi_{2}(x,-z)-\phi_{1}(x, z) .
\end{aligned}
$$

For all other values of the spectral parameter $x \neq x_{m}$, the functions $\phi_{1,2}(x, z)$ are defined via $(5 \mathrm{a}, 5 \mathrm{~b})$ only within the circle $|z+g|<2 g$. Furthermore the eigenfunctions are not normalized when expressed in terms of $(5 a, 5 b)$. Both difficulties are overcome in the following.

\section{TIME EVOLUTION}

If an initial state $\left|\phi_{0},+\right\rangle$ with fixed parity $p=1$ is prepared at time $t=0$, the time evolution takes place within $\mathcal{H}_{+}$and reads

$$
|\phi(t)\rangle=e^{-i H_{+} t}\left|\phi_{0}\right\rangle=\sum_{m} e^{-i E_{m}^{+} t}\left|\psi_{m}\right\rangle \frac{\left\langle\psi_{m} \mid \phi_{0}\right\rangle}{\left\langle\psi_{m} \mid \psi_{m}\right\rangle}
$$

The task consists in computing $\left\langle\psi_{m} \mid \phi_{0}\right\rangle$ and with that the norms $\left\langle\psi_{m} \mid \psi_{m}\right\rangle$ for $m=0,1,2, \ldots$.

\section{A. Formal solution}

Starting from $(5 \mathrm{a}, 5 \mathrm{~b})$ we obtain an expansion of $\left|\psi_{m}\right\rangle$ into normalized eigenstates of the shifted harmonic oscillator,

$$
|n ; g\rangle=\frac{e^{-g^{2} / 2}}{\sqrt{n !}}(z+g)^{n} e^{-g z}
$$

as follows:

$$
\begin{aligned}
& \left|\psi_{m}\right\rangle=e^{g^{2} / 2} \sum_{n=0}^{\infty} \sqrt{n !} K_{n}\left(x_{m}\right)(-1)^{n}|n ;-g\rangle, \\
& \left|\psi_{m}\right\rangle=e^{g^{2} / 2} \sum_{n=0}^{\infty} \sqrt{n !} K_{n}\left(x_{m}\right) \frac{\Delta}{x_{m}-n}|n ; g\rangle .
\end{aligned}
$$

From these equations and the fact that the eigenbasis of the shifted harmonic oscillator $\{|n ; g\rangle\}$ is orthonormal we obtain two equivalent representations of the squared norm of the eigenstates:

$$
\begin{aligned}
\left\langle\psi_{m} \mid \psi_{m}\right\rangle & =e^{g^{2}} \sum_{n=0}^{\infty} n ! K_{n}^{2}\left(x_{m}\right) \\
& =e^{g^{2}} \sum_{n=0}^{\infty} n ! \frac{\Delta^{2}}{\left(x_{m}-n\right)^{2}} K_{n}^{2}\left(x_{m}\right) .
\end{aligned}
$$

Using the expansions of the initial state $\phi_{0}$ into shifted oscillator states $\phi_{0}=\sum_{n=0}^{\infty} \alpha_{n}|n ;-g\rangle=\sum_{n=0}^{\infty} \beta_{n}|n ; g\rangle$ we have

$$
\begin{aligned}
\left\langle\psi_{m} \mid \phi_{0}\right\rangle & =e^{g^{2} / 2} \sum_{n=0}^{\infty} \sqrt{n !} K_{n}\left(x_{m}\right)(-1)^{n} \alpha_{n} \\
& =e^{g^{2} / 2} \sum_{n=0}^{\infty} \sqrt{n !} K_{n}\left(x_{m}\right) \frac{\Delta}{x_{m}-n} \beta_{n} .
\end{aligned}
$$

The preceding equations provide a formal solution of the time evolution problem of (9).

\section{B. Asymptotic numerics}

In practice a numerical calculation of $(12,13)$ fails as the sums diverge for all $x$ which do not exactly coincide with a value $x_{m}$ of the spectrum. For every approximation $\tilde{x}_{m}$ to $x_{m}$, the absolute values of the summands of the series in $(12,13)$ start growing for an $n>n^{\prime}$, with $n^{\prime}$ unknown a priori. In Fig. 1(a), the dashed lines show this behavior for the example of the summands of the norm calculated via (12a). The picture suggests the numerical finding we confirmed for different parameters, that $n^{\prime}$ grows for growing eigenenergies $x_{m}$. We further find numerically that $n^{\prime}$ is large if the error of the approximation $\delta=\left|x_{m}-\tilde{x}_{m}\right|$ is small, which is characteristic for series that are asymptotically valid. It will be shown in the next section that this asymptotic validity holds rigorously true for the series in $(12,13)$.

Due to its asymptotic validity for $\delta \rightarrow 0$, we can evaluate (12a) by approximating

$$
\left\langle\psi_{m} \mid \psi_{m}\right\rangle=e^{g^{2}} \sum_{n=0}^{N} n ! K_{n}^{2}\left(\tilde{x}_{m}\right)+R_{N} .
$$

Here the cutoff $N$ must be chosen large enough so that the relative error $R_{N} /\left\langle\psi_{m} \mid \psi_{m}\right\rangle$, estimated as

$$
\epsilon_{\mathrm{rel}}(N)=\frac{N ! K_{N}^{2}\left(\tilde{x}_{m}\right)}{\sum_{n=0}^{N-1} n ! K_{n}^{2}\left(\tilde{x}_{m}\right)},
$$

is as small as desired. If the evaluation of (15) is done with a low-precision approximation $\tilde{x}_{m}$ of $x_{m}$, the series might start to grow before the precision of $\epsilon_{\text {rel }}$ is achieved, i.e., $n^{\prime}<N$. In this case the calculation has to 


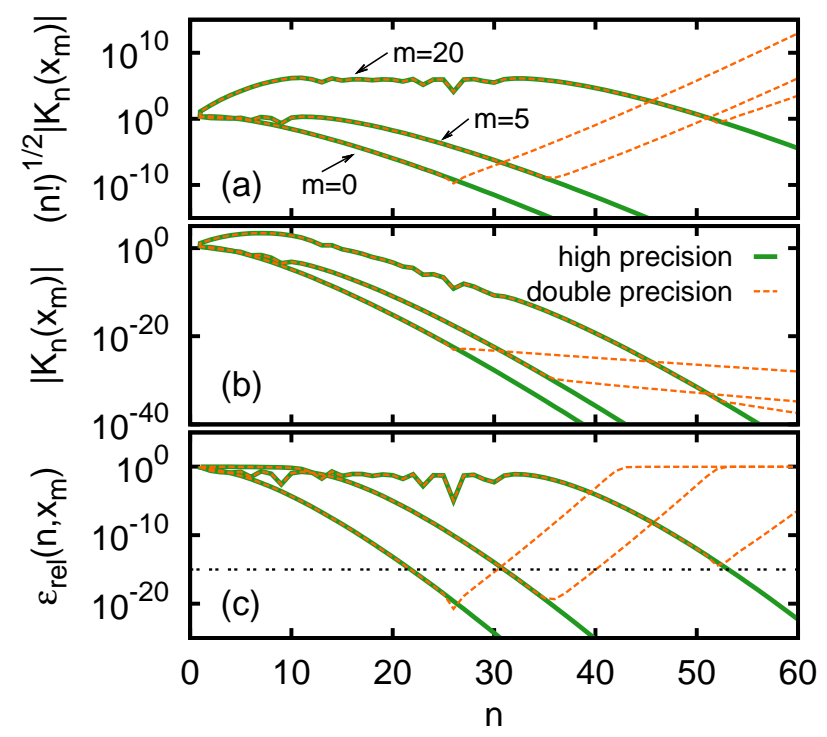

FIG. 1. Panel (a) shows the square root of the absolute values of the summands $\sqrt{n !}\left|K_{n}\left(x_{m}\right)\right|$ of $(12 \mathrm{a}, 14)$. Panel (b) shows the absolute value of the coefficients $\left|K_{n}\left(x_{m}\right)\right|$ (see Eq. (6)) of the eigenfunctions and panel (c) shows the relative error $\epsilon_{\text {rel }}(N=n)(15)$ of the series expression in Eq. (14). All quantities are depicted for three eigenenergies $\left\{x_{0}, x_{5}, x_{20}\right\}$ and the parameters $g / \omega=0.7, \Delta / \omega=0.25$. We present a calculation with double precision and $\delta=10^{-10}$ (dashed lines) and a calculation with high (50 digit) precision and $\delta=10^{-30}$ (solid lines). The horizontal line in panel (c) marks the value $\epsilon_{\mathrm{rel}}=10^{-15}$.

be repeated with a better approximation $\tilde{x}_{m}$, i.e., with a smaller error $\delta$. The expressions (12b) and (13) can be treated analogously.

In Fig. 1 we illustrate the asymptotic behavior of the sum in (12a). We want to evaluate it up to double precision (i.e., by demanding $\epsilon_{\text {rel }}=10^{-15}$ ) which is in general the required precision for possible further computations. We evaluate (12a) for two different values of $\tilde{x}_{m}$. The first value is calculated with double precision and $\delta=10^{-10}$. Although a computation of the norm is possible for the lower part of the spectrum, for the twentieth eigenvalue $x_{20}$ the error $\epsilon_{\text {rel }}$ does not become as small as the desired accuracy $10^{-15}$, but starts to grow before. In a second step we evaluate the norm with an approximation $\tilde{x}_{m}$ obtained in a high (50 digit) precision calculation $\left(\delta=10^{-30}\right)[19]$. This setup is depicted by the solid lines in Fig. 1(c) which cross the horizontal dashed line, marking $\epsilon_{\text {rel }}=10^{-15}$, also for $x_{20}$. However, the coefficients $K_{n}\left(x_{m}\right)$ in panel (b) are almost identical for the highand low-precision calculations for $n<n^{\prime}$, i.e., before $\epsilon_{\text {rel }}$ starts to grow. This observation serves as an a posteriori confirmation of the asymptotic validity of (12a). In the regime where the series is asymptotically meaningful $\left|K_{n}\right| \lesssim \frac{1}{\sqrt{n !}}$ holds, while for $n>n^{\prime},\left|K_{n}\right|$ decays exponentially, which is too slow to compensate the growth of $\sqrt{n !}$. These regimes are separated by clear kinks in Fig. 1(b).

The problems that arise when choosing a low-precision calculation are related to the structure of the three-term recursion (6) that determines the sequence $K_{n}\left(\tilde{x}_{m}\right)$. For high values of $\tilde{x}_{m}$, the absolute value of $K_{n}\left(\tilde{x}_{m}\right)$ becomes so high that the sign-changing behavior of the sequence leads to large numerical errors. For this reason, in a double-precision calculation even the spectrum cannot be determined for $x \gtrsim 40$ as the computation of the function in Eq. (8) becomes numerically unstable. The results of Sec. IV are all calculated using the high-precision setup of Fig. 1.

\section{ABSOLUTELY CONVERGENT REPRESENTATION OF $\phi_{1,2}(x, z)$}

The reason for the divergence of $(12,13)$ is that $\phi_{1,2}(x, z)$ in (5) is ill-defined for $|z+g| \geq 2 g$ if $x \neq x_{m}$. In the following, we present an analytical continuation of $\phi_{1,2}(x, z)$ to the left complex half-plane $\Re(z)<0$ that is well defined for all $x$. Using this analytical continuation, we perform the integration of the scalar product (2) over the full complex plane and obtain finite values for the norm (12) and scalar products (13) also for $x \neq x_{m}$. For $x=x_{m}$ this evaluation yields the values associated to the true eigenstates. A comparison with the results of the preceding subsection then establishes the asymptotic validity of formulae $(12,13)$.

The squared norm $\left\langle\psi_{m} \mid \psi_{m}\right\rangle$ reads

$$
\left\langle\psi_{m} \mid \psi_{m}\right\rangle=\int \frac{\mathrm{d} z \mathrm{~d} \bar{z}}{\pi} e^{-z \bar{z}} \psi\left(x_{m}, \bar{z}\right) \psi\left(x_{m}, z\right) .
$$

To compute (16), one needs $\psi\left(x_{m}, z\right)$ in the whole complex plane. Using the analytical continuation of $\phi_{1}(z), \phi_{2}(z)$ into the left complex half-plane, we define for arbitrary real $x$,

$$
\begin{array}{r}
\langle\psi(x) \mid \psi(x)\rangle=\int_{\text {left } \mathrm{HP}} \frac{\mathrm{d} z \mathrm{~d} \bar{z}}{\pi} e^{-z \bar{z}} \phi_{1}(x, \bar{z}) \phi_{1}(x, z) \\
+\int_{\text {right HP }} \frac{\mathrm{d} z \mathrm{~d} \bar{z}}{\pi} e^{-z \bar{z}} \phi_{2}(x,-\bar{z}) \phi_{2}(x,-z) \\
=\int_{\text {left HP }} \frac{\mathrm{d} z \mathrm{~d} \bar{z}}{\pi} e^{-z \bar{z}}\left(\left|\phi_{1}(x, z)\right|^{2}+\left|\phi_{2}(x, z)\right|^{2}\right) .
\end{array}
$$

The function $\psi(x, z)$, defined by $\phi_{1}(x, z)$ in the left and by $\phi_{2}(x,-z)$ in the right half-plane, exhibits a discontinuity along the imaginary axis if $x-g^{2} \notin \operatorname{spec} H_{+}$, but becomes analytic everywhere if $x=x_{m}$. By replacing (16) by (17), we obtain finite expressions for all $x$ and the correct ones if $x-g^{2}$ belongs to the spectrum.

The analytic continuation of $\phi_{1,2}(x, z)$ from the circle with radius $2 g$ around $z=-g$ to the left half-plane is constructed with a Möbius transformation. The system 


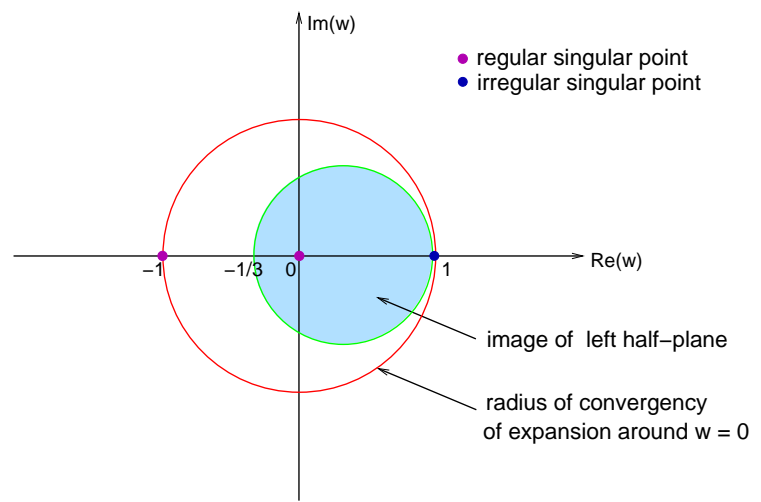

FIG. 2. Image of left $z$ half-plane (light blue) in the $w$ plane using the Möbius transformation (19).

of ordinary differential equations satisfied by $\phi_{1}(z), \phi_{2}(z)$ reads $[16]$

$$
\begin{aligned}
& (z+g) \phi_{1}^{\prime}(z)+\left(g z-x+g^{2}\right) \phi_{1}(z)+\Delta \phi_{2}(z)=0 \\
& (z-g) \phi_{2}^{\prime}(z)-\left(g z+x-g^{2}\right) \phi_{2}(z)+\Delta \phi_{1}(z)=0 .
\end{aligned}
$$

Primes denote differentiation with respect to $z$. The system is characterized by two regular singular points at $z= \pm g$ and one irregular singular point at infinity [20]. Consider now the transformation, $z \rightarrow w(z)$,

$$
w(z)=\frac{z / g+1}{z / g-3}, \quad z(w)=g \frac{3 w+1}{w-1},
$$

which maps the irregular singular point at $z=\infty$ to $w=1$ and the two regular singular points at $z=\mp g$ to $w=0$ and $w=-1$ respectively (see Fig. 2). The left $z$ half-plane is mapped onto the disk with radius $2 / 3$ and center $w=1 / 3$ whereas the imaginary $z$-axis maps onto the boundary of the disk. With $\tilde{x}=4 g^{2}-x$, the system (18) reads in the variable $w$,

$$
\begin{aligned}
& w(w-1) \tilde{\phi}_{1}^{\prime}(w)-\left(\frac{4 g^{2}}{w-1}+\tilde{x}\right) \tilde{\phi}_{1}(w)=\Delta \tilde{\phi}_{2}(w), \\
& \frac{w^{2}-1}{2} \tilde{\phi}_{2}^{\prime}(w)+\left(\frac{4 g^{2}}{w-1}+6 g^{2}-\tilde{x}\right) \tilde{\phi}_{2}(w)=\Delta \tilde{\phi}_{1}(w) .
\end{aligned}
$$

It has regular singular points at $w=-1,0$ and one irregular singular point at $w=1$, while $w=\infty$ is a regular point. Now we may expand the functions $\tilde{\phi}_{j}(w)$ with $j=1,2$ in a power series around the regular singular point $w=0$,

$$
\tilde{\phi}_{j}(x, w)=\sum_{n=0}^{\infty} a_{n}^{(j)}(x) w^{n},
$$

and obtain, with the definition

$$
\boldsymbol{v}_{n+1}=\left(\begin{array}{l}
a_{n+1}^{(2)} \\
a_{n}^{(1)}
\end{array}\right),
$$

for $n \geq-1$ the following four-term matrix recurrence

$$
\boldsymbol{v}_{n+1}=\boldsymbol{A}_{n} \boldsymbol{v}_{n}+\boldsymbol{B}_{n} \boldsymbol{v}_{n-1}+\boldsymbol{C}_{n} \boldsymbol{v}_{n-2} .
$$

The coefficient matrices are given by

$$
\begin{aligned}
\boldsymbol{A}_{n} & =\left(\begin{array}{cc}
\frac{\left(4 g^{2}-2 \tilde{x}+n\right)(n-x)+2 \Delta^{2}}{(n+1)(n-x)} & \frac{2 \Delta}{n+1}\left(1-\frac{\tilde{x}+2 n-2}{n-x}\right) \\
\frac{-\Delta}{n-x} & \frac{\tilde{x}+2 n-2}{n-x}
\end{array}\right), \\
\boldsymbol{B}_{n} & =\left(\begin{array}{cc}
\frac{\left(2 \tilde{x}-12 g^{2}+n-1\right)(n-x)-2 \Delta^{2}}{(n+1)(n-x)} & \frac{2 \Delta(n-2)}{(n+1)(n-x)} \\
\frac{\Delta}{n-x} & \frac{2-n}{n-x}
\end{array}\right), \\
\boldsymbol{C}_{n} & =\left(\begin{array}{ll}
\frac{2-n}{n+1} & 0 \\
0 & 0
\end{array}\right) .
\end{aligned}
$$

The condition $\phi_{j}(x, z)=\tilde{\phi}_{j}(x, w(z))$ fixes the initial values of $\boldsymbol{v}_{0}$ and $\boldsymbol{v}_{1}$, namely

$$
\begin{aligned}
& a_{0}^{(2)}=e^{g^{2}}, \quad a_{0}^{(1)}=\frac{\Delta}{x} e^{g^{2}}, \\
& a_{1}^{(2)}=2\left(x-2 g^{2}-\frac{\Delta^{2}}{x}\right) e^{g^{2}} .
\end{aligned}
$$

Together with $\boldsymbol{v}_{-1}=\mathbf{0}$, all $\boldsymbol{v}_{n}$ for $n \geq 2$ follow recursively.

The asymptotic behavior of the $a_{n}^{(j)}$ can be inferred from (23) as $\lim _{n \rightarrow \infty}\left|a_{n+1}^{(j)} / a_{n}^{(j)}\right|=1$. Hence the radius of absolute convergence of the expansion (21) is 1 for all $x$, as expected from the singularity structure of (20): The expansion around $w=0$ converges up to the neighboring singular points, $w= \pm 1$. Now we can perform the integral in (17) because the image of the left half-plane is contained entirely within the region of absolute convergence of the series expansion (21), which furnishes the desired analytical continuation of the functions $\phi_{j}(x, z)$. The integral (17) reads in $w$ coordinates

$$
\begin{aligned}
\langle\psi(x) \mid \psi(x)\rangle=\int_{\text {disk }} & \frac{\mathrm{d} w \mathrm{~d} \bar{w}}{\pi} \frac{4 g^{2}}{|w-1|^{4}} e^{-z(w) z(\bar{w})} \\
& \times\left(\left|\tilde{\phi}_{1}(x, w)\right|^{2}+\left|\tilde{\phi}_{2}(x, w)\right|^{2}\right) .
\end{aligned}
$$

The numerical integration in (26) is done in polar coordinates over the disk in the $w$ plane obtained by mapping the left half-plane with the Möbius transformation (19) (see Fig. 2). Fig. 3 shows that the integrand in (26) is a smooth function, for which the numerical integration is easy to control. The singularity that is still present in the wave function $\tilde{\phi}_{j}(x, w)$ for $w=1$ is suppressed in the integrand by the regularizing factor $e^{-z(w) z(\bar{w})}$. As expected from the convergence properties of $\tilde{\phi}_{j}(x, w)$, the representation of the norm in Eq. (26) depends smoothly on the argument $x$. This means that for values of $x$ that differ only slightly from the spectral values used in Fig. 3, the corresponding plots of the integrand are essentially the same.

Finally, we confirmed the validity of the asymptotic formula (14) by comparing it to a numerical integration of $(17)$. The validity of the remaining expressions $(12,13)$ was also checked. 


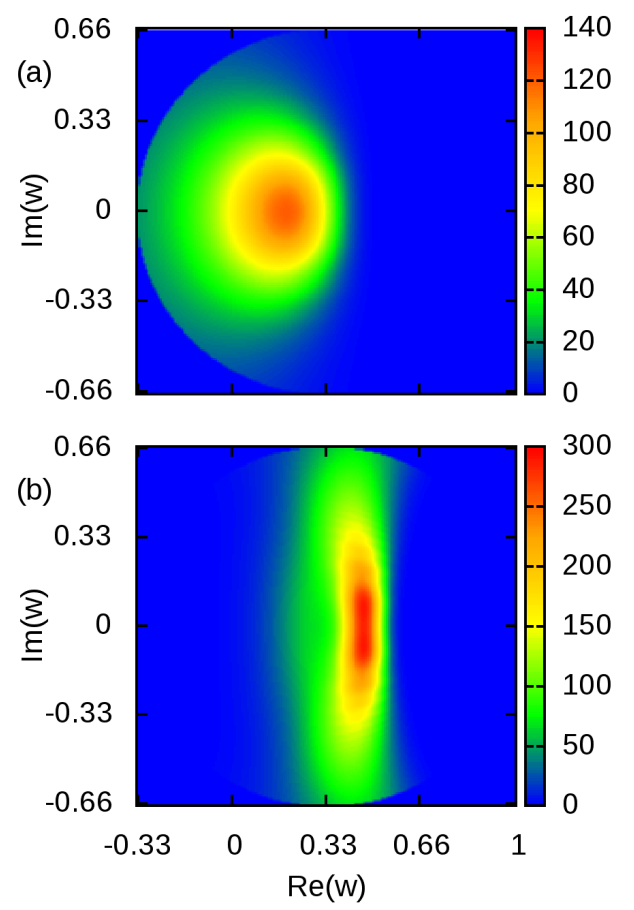

FIG. 3. The integrand of (26) for the first (a) and the fifth (b) eigenenergy $\left(x_{0}=0.06038 \ldots\right.$ and $\left.x_{5}=4.9355 \ldots\right)$ in the positive parity subspace for parameters $g / \omega=0.7$ and $\Delta / \omega=$ 0.25 .

\section{SPIN OBSERVABLES}

In this section we calculate the time evolution of $\left\langle\sigma_{z}(t)\right\rangle$ and $\left\langle\sigma_{x}(t)\right\rangle$ using the method presented in Sec. II. The "population inversion" $\sigma_{z}(t)$ has been thoroughly investigated for the resonant case $(g / \omega \ll 1$ and $\Delta / \omega \simeq 0.5)$ within the rotating-wave approximation, where it shows the typical collapse and revival oscillations [21] as well as within analytical [14] and numerical [22] treatments of the full model. Here we follow a different path by investigating the problem for the non-resonant case and arbitrary parameters in all coupling regimes. We find a qualitatively different behavior for $\left\langle\sigma_{z}(t)\right\rangle$ and $\left\langle\sigma_{x}(t)\right\rangle$ for strong coupling. As will be discussed below, this difference can be understood in terms of the $\mathbb{Z}_{2}$ symmetry, manifested in the two invariant parity chains.

\section{A. Time evolution of $\left\langle\sigma_{z}\right\rangle$}

To study the time evolution of $\sigma_{z}$, we prepare the system at time $t=0$ in a product of a coherent state $|\alpha\rangle=e^{-\alpha^{2} / 2+\alpha z}$ with an eigenstate of $\sigma_{z}$

$$
|\psi(0)\rangle=|\alpha\rangle \otimes|+1\rangle
$$

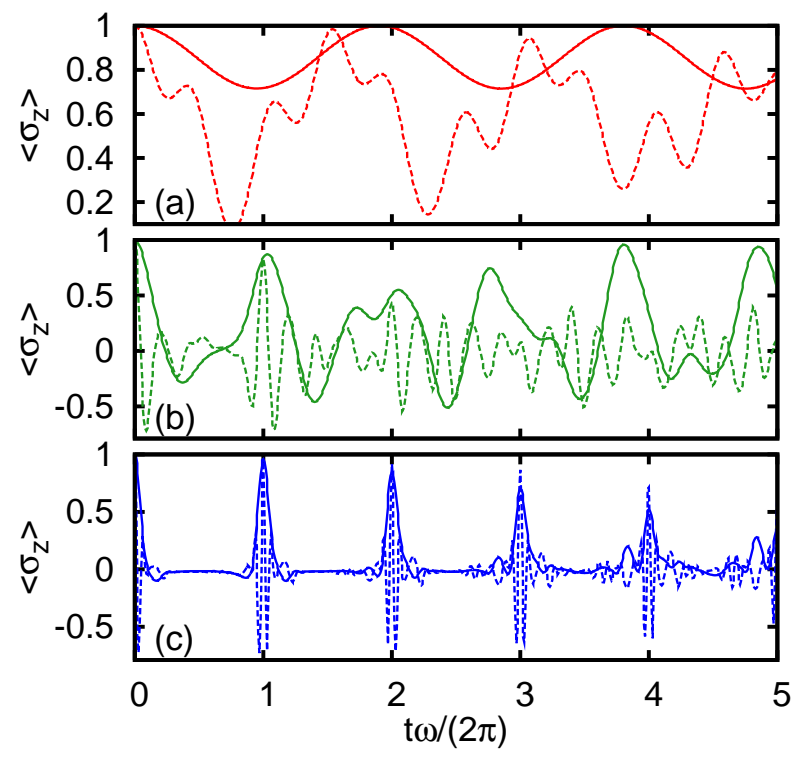

FIG. 4. $\left\langle\sigma_{z}(t)\right\rangle$ for $\Delta / \omega=0.25$ and (a) $g / \omega=0.1$, (b) $g / \omega=$ 0.7 , (c) $g / \omega=2$. The evolution starts with coherent states with $\alpha=0$ (solid line) and $\alpha=2$ (dashed line).

In terms of parity chains it reads,

$$
|\psi(0)\rangle=e^{-\alpha^{2} / 2}(|\operatorname{ch}(\alpha z),+\rangle+|\operatorname{sh}(\alpha z),-\rangle) .
$$

Using the isomorphism (3), we find for the time evolution of $\left\langle\sigma_{z}\right\rangle$

$$
\begin{aligned}
\left\langle\sigma_{z}(t)\right\rangle= & e^{-\alpha^{2}}\left[\left\langle\cosh (\alpha z)\left|e^{-i H_{+} t} \hat{T} e^{i H_{+} t}\right| \cosh (\alpha z)\right\rangle\right. \\
& \left.-\left\langle\sinh (\alpha z)\left|e^{-i H_{-} t} \hat{T} e^{i H_{-} t}\right| \sinh (\alpha z)\right\rangle\right],
\end{aligned}
$$

where $\hat{T}$ is the reflection operator in $\mathcal{B}$. This expectation value is plotted in Fig. 4. As can be seen from (29) the time evolution is solely governed by the commutator $\left[\hat{T}, H_{ \pm}\right]$and the system evolves separately in each parity subspace. Thus, when evaluating the expression, one can assign a definite parity to the frequencies $E_{n}^{ \pm}-E_{m}^{ \pm}$. As these frequencies are always of order $(n-m) \omega$ for sufficiently strong coupling $g / \Delta$, the behavior on time scales of a few $2 \pi / \omega$ is dominated by $\omega$ as the smallest frequency.

\section{B. Time evolution of $\left\langle\sigma_{x}\right\rangle$}

In complete analogy to the case of $\sigma_{z}$, we prepare the system at time $t=0$ in a product of a coherent state with an eigenstate of $\sigma_{x}$

$$
|\psi(0)\rangle=|\alpha\rangle \otimes \frac{1}{\sqrt{2}}(|+1\rangle+|-1\rangle) .
$$




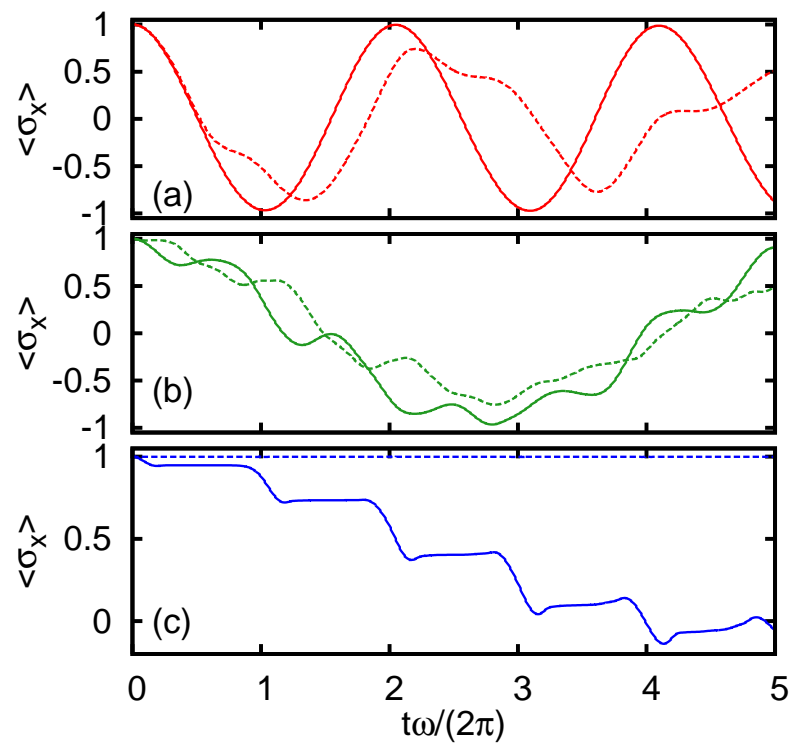

FIG. 5. $\left\langle\sigma_{x}(t)\right\rangle$ for the same setup as in Fig. 4. Note that (c) shows that $\left\langle\sigma_{x}(t)\right\rangle \simeq 1$ for all times if the initial state is given by the linear combination of two cat states in Eq. (32). We observe a sharp step-like decay (solid line) in (c) for a system prepared in the vacuum $(\alpha=0)$. For a system prepared in a coherent state with $\alpha=g / \omega=2$, a constant plateau is seen in (c) (dashed line).

Using the representation in the two parity subspaces, we obtain the time evolution of $\left\langle\sigma_{x}\right\rangle$ as

$$
\left\langle\sigma_{x}(t)\right\rangle=\frac{1}{2}\left\langle\alpha\left|e^{-i H_{+} t} e^{i H_{-} t}+e^{-i H_{-} t} e^{i H_{+} t}\right| \alpha\right\rangle .
$$

This expectation value is plotted in Fig. 5. When evaluating the expression in (31), the frequencies have the form $E_{n}^{-}-E_{m}^{+}$with eigenenergies from different parity chains. These frequencies may become arbitrary small. In particular the frequency $E_{n}^{+}-E_{n}^{-}$is small for strong coupling, because $E_{n}^{+}$and $E_{n}^{-}$both approach the same value $n \omega-g^{2} / \omega$ for growing $g / \Delta$ from above and below. Therefore small frequencies dominate $\left\langle\sigma_{x}(t)\right\rangle$ for sufficiently strong coupling. We note that the frequency $E_{n}^{+}-E_{n}^{-}$vanishes if the parameters $g$ and $\Delta$ satisfy the Juddian condition $K_{n}(n \omega)=0$.

For $g / \omega=2$ and $\Delta / \omega=0.25$ (i.e., in the so-called deep strong coupling regime [18]) we observe two features in Fig. 5(c). The first is the sharp step-like decay of $\left\langle\sigma_{x}(t)\right\rangle$ for a system prepared in the vacuum $(\alpha=0)$ at $t=0$. Each step has the length $2 \pi / \omega$. This structure disappears for higher values of $\alpha$.

Secondly, $\left\langle\sigma_{x}(t)\right\rangle$ stays perfectly constant for $\alpha=g / \omega$ as depicted by the dashed line in Fig. 5(c). In this case, the representation of the initial state (30) in the parity subspaces is a superposition of the two "Schrödinger cat states" $\left|C_{ \pm}\right\rangle \in \mathcal{H}_{ \pm}[23]$

$$
\begin{aligned}
& |\Psi(0)\rangle=\frac{1}{\sqrt{2}}\left(\left|C_{+}\right\rangle+\left|C_{-}\right\rangle\right) \text {for } \alpha=\frac{g}{\omega}, \\
& \left|C_{+}\right\rangle=e^{-g^{2} / 2 \omega^{2}}(\operatorname{ch}(g z / \omega) \otimes|+1\rangle+\operatorname{sh}(g z / \omega) \otimes|-1\rangle), \\
& \left|C_{-}\right\rangle=e^{-g^{2} / 2 \omega^{2}}(\operatorname{ch}(g z / \omega) \otimes|-1\rangle+\operatorname{sh}(g z / \omega) \otimes|+1\rangle) .
\end{aligned}
$$

The states $\left|C_{ \pm}\right\rangle$are eigenstates of $H_{ \pm}$for $\Delta=0$ and one would expect their approximate conservation over time for small $\Delta$ but decay at longer time scales or appreciable ratio $\Delta / g$. It is therefore surprising that the dynamical protection of $\left|C_{ \pm}\right\rangle$(and concomitantly $\sigma_{x}$ ) extends to long times (see next section) and applies to quite large $\Delta / g$, which is 0.125 in Figs. 5(c) and $7(\mathrm{c})$. This remarkable feature of the QRM at couplings $g / \omega>1$ is related to the excellent approximation of the true ground state in each parity chain by the shifted vacuum state (see Eq. (10)) and will be discussed in more detail elsewhere. The dynamical protection of $|\Psi(0)\rangle$ leads to the constant plateau (dashed line) in Fig. 5(c). Fig. 7(c) shows that this behavior persists even on long time scales.

\section{Long-time behavior}

In the preceding subsections we pointed out that for sufficiently high $g / \Delta$ the frequencies appearing in the time evolution of $\left\langle\sigma_{z}(t)\right\rangle$ have an approximate lower bound of $\omega$ while this is not the case for $\left\langle\sigma_{x}(t)\right\rangle$. This is inferred from an analysis of the representation in the parity subspaces and can be seen by comparing Figs. 4(b),(c) with Figs. 5(b),(c). Only in the latter slow oscillations are visible.

This effect is also observed in the long-time behavior displayed in Figs. 6 and 7. For weak coupling $(g / \omega=0.1)$ the time evolution of $\left\langle\sigma_{z}(t)\right\rangle$ and $\left\langle\sigma_{x}(t)\right\rangle$ is rather similar in Figs. 6(a) and 7(a). For strong coupling $(g / \omega \geq 0.7)$ the same analysis as for short time scales applies. In the case of $\sigma_{z}, \omega$ remains the smallest frequency and the oscillations are dominated by higher harmonics, although a modulation by small beat frequencies, due to the superposition of contributions from both parity subspaces (29), is visible. By contrast, the overall behavior of $\left\langle\sigma_{x}(t)\right\rangle$ for $g / \omega \geq 0.7$ is dominated by oscillations with period $T^{*}>2 \pi / \omega$, as seen in Fig. $7(\mathrm{~b})$ and (c). According to the argument of the previous subsection, $T^{*}$ grows with $g$. For $g / \omega=0.7$ the dominant frequency $1 / T^{*}$ is then essentially the same for initial coherent states with $\alpha=0$ and $\alpha=2$, see Fig. 7(b). This is true also for the case of $g / \omega=2$, where it is seen that the step-like structures follow an overall oscillation with period $T^{*}$. Only in the special case of $\alpha=g / \omega$, in which the time evolution is dynamically protected, the initial expectation value is maintained for arbitrary long times (dashed line in Fig. 7(c)). 


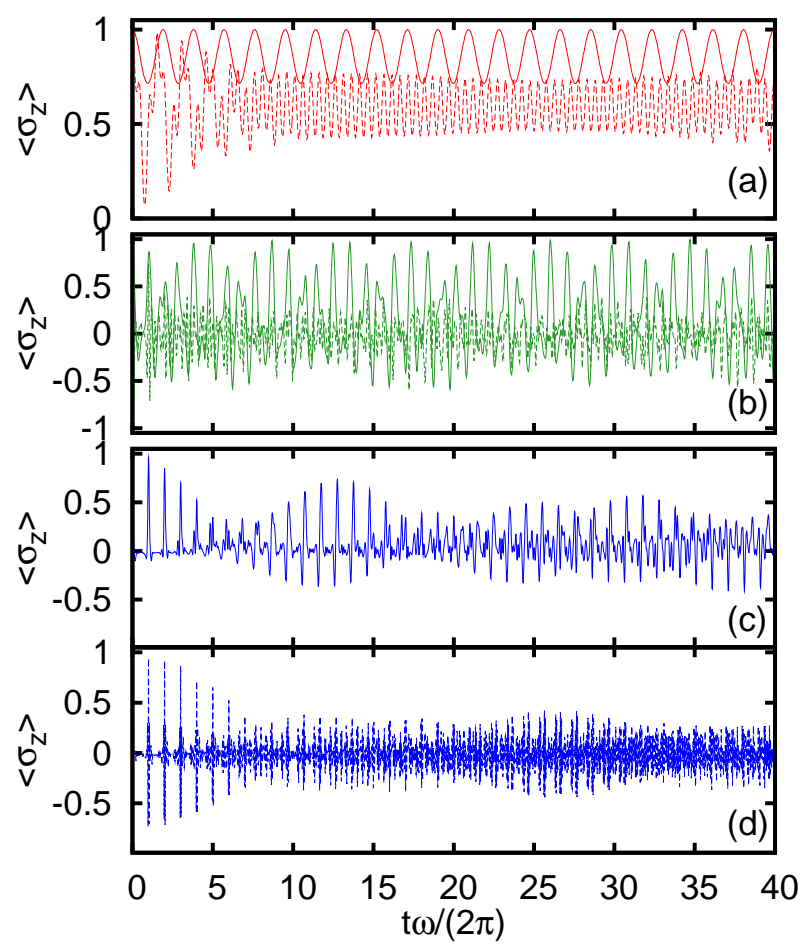

FIG. 6. $\left\langle\sigma_{z}(t)\right\rangle$ for times up to 40 fundamental periods $\left(T_{0}=\right.$ $2 \pi / \omega)$ and the same parameters as in Fig. 4. The case $g / \omega=2$ is split into panels (c), $\alpha=0$, and (d), $\alpha=2$, for clarification. The effect of the modulating beat frequencies is not visible for $g / \omega=0.1$ on this time scale, whereas it appears clearly for $g / \omega=0.7$ and 2 . In all three cases higher harmonics dominate the time evolution for $\alpha=2$ (dashed lines in (a) and (b), resp. $\operatorname{panel}(d))$.

\section{CONCLUSION}

We have described a method to compute exactly all eigenstates of the quantum Rabi model together with their norms and scalar products with states of the standard basis. In this way the development of an arbitrarily prepared initial state can be obtained for short and long times. The method uses the $\mathbb{Z}_{2}$ invariance of the model, the representation of the oscillator degree of freedom in the Bargmann space of analytical functions and a conformal mapping to obtain absolutely convergent expansions of the norms and overlaps with Fock states. The method overcomes the limitations of numerical procedures or analytical approximations based on a truncated Hilbert space. Moreover, these techniques themselves can now be mathematically justified by comparison with the analytical solution and retain in this way their usefulness for practical calculations. This will be discussed elsewhere. The splitting of the full space into two invariant subspaces results in a qualitatively different behavior of $\left\langle\sigma_{z}(t)\right\rangle$ and $\left\langle\sigma_{x}(t)\right\rangle$ on intermediate and long time scales:

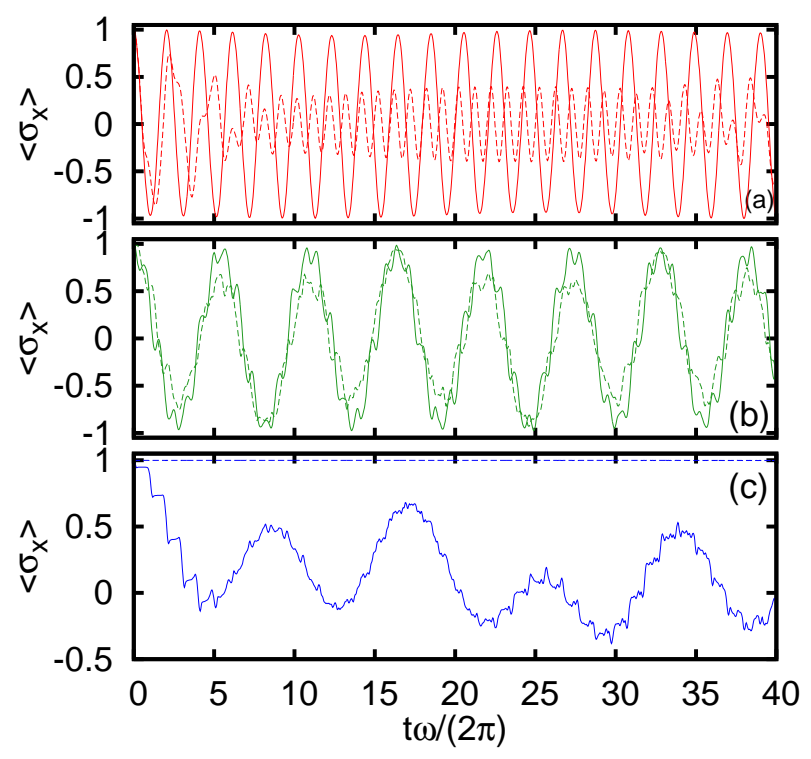

FIG. 7. $\left\langle\sigma_{x}(t)\right\rangle$ for times between 0 and $40 T_{0}$ as in Fig. 6 and parameters as in Fig. 5. The qualitative behavior depends clearly on the initial state for small coupling $g / \omega=0.1$ (top panel) but not for $g / \omega=0.7$ (b). The dominating period $T^{*}>T_{0}$ grows with $g$ due to decreasing differences $E_{n}^{+}-E_{n}^{-}$. For $\alpha=g / \omega=2,\left\langle\sigma_{x}\right\rangle$ stays constant.

$\left\langle\sigma_{z}(t)\right\rangle$ is always dominated by integer multiples of the photon frequency $\omega$. These oscillations are modulated by small beat frequencies because the QRM spectrum is not equidistant and both parity chains interfere. $\left\langle\sigma_{x}(t)\right\rangle$, on the other hand, shows oscillations with a dominant frequency much smaller than $\omega$, which is directly related to the mixing of both parity chains effected by the operator $\sigma_{x}$. The oscillation period grows with growing coupling between qubit and radiation field. For initial coherent states fulfilling the condition (32), we observe "dynamical protection" in the deep strong coupling regime which could be relevant to the control of fast quantum gates within circuit QED.

\section{ACKNOWLEDGMENTS}

This work was supported in part by Deutsche Forschungsgemeinschaft through TRR 80.
[1] L. Allen, J. H. Eberly, Optical Resonance and Two Level Atoms, Wiley N.Y. (1975).
[2] H. Rongsheng, L. Zijing, W. Kelin, Phys. Rev. B 65, 174303 (2002). 
[3] T. Niemczyk, F. Deppe, H. Huebl, E. P. Menzel, F. Hocke, M. J. Schwarz, J. J. Garcia-Ripoll, D. Zueco, T. Hümmer, E. Solano, A. Marx, and R. Gross, Nature Physics 6, 772 (2010).

[4] P. Forn-Díaz, J. Lisenfeld, D. Marcos, J. J. García-Ripoll, E. Solano, C. J. P. M. Harmans, and J. E. Mooij, Phys. Rev. Lett. 105, 237001 (2010).

[5] T. Pellizzari, S. A. Gardiner, J. I. Cirac, P. Zoller, Phys. Rev. Lett. 75, 3788 (1995).

[6] S. Schweber, Ann. Phys. (N.Y.) 41, 205 (1967).

[7] H. G. Reik, H. Nusser, and L. A. Amarante Ribeiro, J. Phys. A 15, 3491 (1982).

[8] H. G. Reik and M. Doucha, Phys. Rev. Lett. 57, 787 (1986).

[9] N. Klenner, J. Weiss, and M. Doucha, J. Phys. C 19, 4673 (1986).

[10] A. Pereverzev and E. R. Bittner, Chem. Phys. 8, 1378 (2006).

[11] T. Liu, K. L. Wang, and M. Feng, EPL 86, 54003 (2009).
[12] F. Pan, X. Guan, Y. Wang, and J. P. Draayer, J. Phys. B 43, 175501 (2010).

[13] J. Hausinger and M. Grifoni, Phys. Rev. A 82, 062320 (2010)

[14] I. D. Feranchuk, L. I Komarov, and A. P. Ulyanenkov, J. Phys A 29, 4035 (1996); I. D. Feranchuk and A. V. Leonov, Phys. Lett. A 373, 4113 (2009); 375, 385 (2011).

[15] V. V. Albert, G. D. Scholes, and P. Brumer, Phys. Rev. A 84, 042110 (2011).

[16] D. Braak, Phys. Rev. Lett. 107, 100401 (2011).

[17] V. Bargmann, Comm. Pure. Appl. Math. 14, 187 (1961).

[18] J. Casanova, G. Romero, I. Lizuain, J. J. García-Ripoll, and E. Solano, Phys. Rev. Lett. 105, 263603 (2010).

[19] We use GiNaC and CLN, http://www.ginac.de.

[20] E. L. Ince, Ordinary Differential Equations, Dover N.Y. 1956.

[21] N. B. Narozhny, J. J. Sanchez-Mondragon, and J. H. Eberly, Phys. Rev. A 23, 236 (1981).

[22] J. Larson, Phys. Scr. 76, 146 (2007).

[23] M. Brune, S. Haroche, J. M. Raimond, L. Davidovich, and N. Zagury, Phys. Rev. A 45, 5193 (1992). 